\title{
Orientación laboral: Una revisión bibliográfica de su conceptualización y su aporte a la persona trabajadora y a las organizaciones laborales
}

\author{
Career Counseling: A Bibliographical Framework and Contribution for Employment \\ Companies and Employees
}

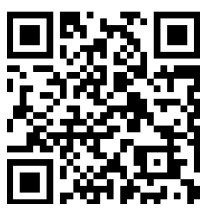

\author{
Josué Jiménez Ulate \\ Universidad Nacional \\ Heredia, Costa Rica \\ josue.jimenez.ulate@una.cr
}

Recibido 1 de febrero de 2016 • Corregido 22 de marzo de 2017 • Aceptado 27 de abril de 2017

\begin{abstract}
Resumen: En la siguiente revisión se realiza una reflexión teórica sobre las transformaciones que experimentan las organizaciones laborales y el trabajo en el siglo XXI, seguidamente se procede a brindar un acercamiento conceptual a la orientación laboral desde el pensamiento sistémico, que permita proponer un marco de acción para los profesionales y las profesionales de esta especialización y los posibles contextos desde los que pueden desarrollar sus intervenciones. Se concluye que el eje central de toda intervención desde la orientación laboral es el ser humano, por lo que se busca promover las competencias para el desarrollo y bienestar laboral de la persona trabajadora desde los diferentes entornos en la que esta interactúa y, a la vez, apoyar a las organizaciones a ser lugares más productivos y con sentido humano por medio de la creación de las condiciones en los entornos laborales que faciliten ese desarrollo, y bienestar personal y de la comunidad.
\end{abstract}

Palabras claves: Orientación laboral; procesos sociolaborales; desarrollo y bienestar laboral; complejidad, pensamiento sistémico.

\begin{abstract}
In the following review, a theoretical reflection is made upon the transformations experienced by the labour organizations and work in the XXI century; this is followed by a conceptual approach to the labour counseling, offered from a systemic thinking perspective that allows to propose an action framework for the discipline specializartion professionals and for the possible contexts from which they can develop interventions. It is concluded that the aim of an intervention from a career counseling perspective will always be the human being; indeed, this field of study targets the promotion of the competencies for the work development and the wellbeing of people within the different contexts in which they interact. At the same time, it is expected to support labor organizations, so that they become more productive places with larger human responsibility, as they enhance the necessary conditions for development and wellbeing of the person and the community in general.
\end{abstract}

Key words: career counseling; social and labor processes; development and labor welfare; complexity; systemic thinking.

${ }^{1}$ Egresado de la Maestría en Orientación Laboral en la Universidad de Costa Rica. Obtuvo su Licenciatura en Orientación en la Universidad Nacional, Costa Rica. Actualmente es académico en la Universidad Nacional, Costa Rica y laboró como orientador en la oficina de Capacitación y Empleabilidad de la Municipalidad de Alajuela. 
doi: http://dx.doi.org/10.15359/ree.21-2.19

URL: http://www.una.ac.cr/educare

CORREO: educare@una.cr

\section{Introducción}

La formación de personas profesionales en orientación laboral inicia recientemente en el país. Esta nueva área de especialización de la orientación tiene como propósito el desarrollo y bienestar de las personas trabajadoras en su contexto sociolaboral. No hay duda de que se ha dado una creciente conciencia de la complejidad de los procesos que ocurren en el mundo del trabajo, además, este ocupa un lugar trascendental en el desarrollo del ser humano y del entorno; pues tal como lo indica la Fundación Demuca (2011), por medio del trabajo, el ser humano "se integra socialmente, además es una fuente de dignificación y autoestima" (p. 10) $y$, a su vez, por medio del trabajo las personas interactúan con las organizaciones laborales y viceversa, contribuyendo a la construcción de los entornos donde ocurren estas interacciones .

Esta circunstancia representa un reto para las futuras generaciones de profesionales en orientación laboral, el cual consiste en la producción de un bagaje de saberes que permitan el análisis y reflexión en torno a las problemáticas actuales que se plantean en este campo del conocimiento.

Por lo tanto, en este artículo, se realiza una reflexión teórica sobre las transformaciones que experimentan las organizaciones laborales y el trabajo en el siglo XXI, seguidamente se procede a brindar un acercamiento conceptual a la orientación laboral desde el pensamiento sistémico, que permita proponer un marco de acción para los grupos profesionales de esta especialización y sus posibles contextos desde los que pueden desarrollar sus intervenciones.

\section{Las organizaciones laborales y el trabajo}

El estudio de la evolución de las organizaciones laborales y del trabajo ha sido abordado "desde varias disciplinas como la Economía, la Historia, la Antropología, la Psicología, etc." (Marín, 2011, p. 28), a partir de las influencias de las condiciones sociales, económicas, políticas, culturales y tecnológicas imperantes.

Estas influencias han producido una serie de transformaciones permanentes en las actividades sociolaborales. Sin embargo, en la actualidad, a diferencia de las otras épocas, estas se han producido en lapsos más cortos. Alcover, Martínez, Rodríguez y Domínguez (2004, p. 491) mencionan que:

La velocidad con la que suceden [las tranformaciones], la acelaración que se imprime a todas las esferas de la actividad humana, la gran rapidez con la que conocimientos, datos, herramientas, sistemas, acontecimientos, ideas, regímenes, ídolos, etc., se suceden y quedan rápidamente obsoletos, desfasados, inútiles, olvidados, e, incluso, desconocidos, todo ello en el relativamente breve periodo de una sola generación. 
Debido a esta velocidad con la que se han impregnado estas transformaciones, cabe cuestionar si las organizaciones laborales han logrado adaptarse a estas o están en proceso; $y$, a partir de ello, comprender la concepción y el papel de la orientación laboral por medio de la reflexión sobre aspectos como la sociedad del conocimiento, las organizaciones y el trabajo en contextos complejos y la empleabilidad.

Desde finales del siglo XX y principios del presente, las economías industriales se han ido transformando en otras, fundadas en el conocimiento (David y Foray, 2002), es decir, en el establecimiento de comunidades que producen y reproducen saberes (en otras palabras, comercializan esos conocimientos); para ello, se requiere que los países con este tipo de economía realicen una alta inversión en educación, investigación, desarrollo de tecnologías de la información y comunicación, entre otros, que permitan fortalecer la calidad de su fuerza laboral.

Estos mismos autores, señalan que "estas comunidades [del saber] se extienden a las organizaciones e instituciones clásicas (las empresas, los centros de investigación, los organismos públicos y gubernamentales) y los miembros de estas [organizaciones] son también los empleados de esas organizaciones" (David y Foray, 2002, p.16). Por esto, las organizaciones laborales en el contexto de la sociedad del conocimiento enfrentan una serie de cambios cuantitativos y cualitativos en sus estructuras, en su producción, así como en las vinculaciones con otras organizaciones en consecuencia de las tecnologías de la información y comunicación. Además, se dan cambios en la relación que esta establece con las personas y generan diferentes formas contractuales y mayor visibilidad de otras modalidades de trabajo distinta al empleo. Por estas circunstancias, Guzmán (2007, p. 130) explica que:

El creciente aumento de la complejidad del mundo nos enfrenta a una realidad cada vez más caótica, plagada de innovaciones tecnológicas que se entremezclan con el uso desenfrenado de los recursos naturales. La tecnología, en este contexto global y voraz opera como agente catalizador que acelera el fenómeno de la complejidad y enfrenta a la humanidad con un nuevo período histórico.

Una visión más reciente de esta realidad descrita, basada en un modelo económico del conocimiento, globalizado e interdependiente, es la que aporta el pensamiento complejo, el cual promueve un conocimiento desde lo global, el contexto, lo multidimensional y lo complejo de los diferentes sistemas construidos por la humanidad (Morin, 1999). Por lo que se requiere de nuevos acercamientos epistemológicos sobre las organizaciones laborales, entre ellos el brindado por el pensamiento complejo, para un análisis más acorde de los procesos que ocurren en estas a partir de las características de la sociedad actual. 
doi: http://dx.doi.org/10.15359/ree.21-2.19

URL: http://www.una.ac.cr/educare

CORREO: educare@una.cr

Las organizaciones laborales, desde una visión del pensamiento complejo, se conciben como "un organismo viviente, [que] se auto-organiza y realiza su auto-producción, al mismo tiempo realiza la auto-eco-organización y la auto-eco-producción" (Morin, 1990, p. 124). Esta visión permite comprender que las organizaciones laborales por medio de la producción de sus bienes, productos y servicios se autoconstruyen y autoorganizan, a la vez que contribuyen a la organización y construcción de los contextos en las que se encuentran inmersas.

Por ello, las organizaciones laborales tienen la necesidad de fases de orden y desorden, intermediados por fases de reordenamiento de sus dinámicas para que den existencia a la organización laboral en el tiempo. Para Morin (1990), el orden "es todo aquello que es repetición, constancia, invariabilidad, todo aquello que puede ser puesto bajo la égida de una relación altamente probable, encuadrado bajo la dependencia de una ley" (p. 125), mientras el desorden "es todo aquello que es irregular, desviación con respecto a una estructura dada, elemento aleatorio, imprevisibilidad" (p. 126). Por lo que la capacidad de innovación se convierte en el elemento que conduce a la existencia y permanencia de esos sistemas, la cual surge en la interacción orden-desorden.

Por tanto, las interacciones entre las fases de orden y desorden dentro del mercado laboral contribuyen a que este alcance un nuevo equilibrio homeostático por medio de la autoecoorganización del mercado laboral, promoviendo en la sociedad periodos de crecimiento económico.

Además, autores como Gallardo (2002) y Alcover et al. (2004) concuerdan en que ante esta comprensión de la realidad, las organizaciones están dando paso de estructuras rígidas y jerárquicas a estructuras orgánicas y flexibles, que les permitan su autoorganización y responder a diversos objetivos laborales, diversa clientela (externa e interna) y diversos contextos.

No obstante, estas circunstancias en las organizaciones laborales han producido una serie de transformaciones en la concepción del trabajo, así como en la referencia de la construcción psicosocial de las personas, pues como lo mencionan Rentería, García, Restrepo y Riascos (2007, p. 140), el trabajo es"algo dinámico, [y] que se configura y reconfigura en la interacción constante entre individuos, organizaciones, instituciones y demás actores sociales que integran dichos escenarios". De esto se podría inferir que en el trabajo intervienen diversos actores sociales como la familia, el sistema educativo, las organizaciones, entre otros; los cuales transmiten, por medio de procesos de socialización, valores para el trabajo, enmarcados dentro del contexto histórico-cultural imperante.

El trabajo ha tenido diversos significados a lo largo de la humanidad; sin embargo, en la actualidad este se puede vincular a diversos aspectos como la capacidad de motivación de las personas, por medio de la satisfacción razonable de sus necesidades 
básicas, necesidades de seguridad, necesidades de pertenencia, necesidades de estima y necesidades de autorrealización (Maslow, 1991). Además, se considera que la dimensión laboral en la carrera de las personas favorece la construcción de estas como sujetos sociales, con la capacidad de transformar sus contextos.

En esta misma linea de pensamiento, Alcover et al. (2004) consideran el trabajo como una actividad transformadora y productiva propia del ser humano, la cual se enmarca en contextos específicos donde se involucran las relaciones, interacciones e intercambios de personas para la producción de bienes y servicios; así como la construcción de sus identidades y subjetividades (Alcover et al., 2004). No obstante, debido a las transformaciones tecnológicas imperantes de la sociedad, las personas requieren nuevas formas de comunicación e interacciones en contextos laborales virtualizados, los cuales son influenciados por la constante diada orden-desorden, a la que las personas han de enfrentarse.

Además, el trabajo enmarcado en estas transformaciones se caracteriza por una mayor flexibilidad, por una disminución de la estabilidad laboral y por la mayor visibilidad de otras modalidades de trabajo distintas al empleo como lo son: los servicios profesionales, la subcontratación, el cooperativismo (Rentería, 2008). Este mismo autor menciona que estas modalidades de trabajo han estado presentes a lo largo de la historia del trabajo, pero que difieren a la forma contractual tradicional de este, es decir, a la relación que se establece entre las organizaciones laborales y las personas; así como a la forma de la prestación de los servicios, el espacio físico-temporal de esta prestación y de compensación (Rentería, 2008).

Asimismo, Sánchez (2003) menciona que otra característica consiste que en el transcurso del tiempo algunas ocupaciones desaparecerán, a la vez que otras aparecerán como parte de las nuevas exigencias de la sociedad del siglo XXI, por lo cual, las personas se podrían beneficiar de los procesos de orientación laboral en el desarrollo y gestion de sus carreras a lo largo de la vida.

Debido a ello, las personas se ven inmersas en procesos de aprendizaje a lo largo de sus vidas, promoviendo el desarrollo de competencias sociolaborales, que les permitan movilizarse dentro de sus contextos sociales. Por ello, la educación basada en competencias debe dar"prioridad a la formación de personas integrales con compromiso ético, que busquen su autorrealización, que aporten al tejido social y que, además, sean profesionales idóneos y emprendedores" (Tobón, 2008, p. 3). Desde esta perspectiva, la educación no solo busca una formación para el trabajo, sino que busca el empoderamiento de las personas y el desarrollo de su criticidad con la finalidad de promover transformaciones en sus realidades.

No obstante, las organizaciones laborales han manifestado dificultades en los procesos de gestión del talento humano, los cuales comprenden los esfuerzos de las organizacionaes laborales para la atracción, retención y desarrollo de las personas trabajadoras, debido a que no 
doi: http://dx.doi.org/10.15359/ree.21-2.19

URL: http://www.una.ac.cr/educare

CORREO: educare@una.cr

se cumplen con las expectativas del sector empresarial, referentes a la calidad del desempeño por parte de sus colaboradores, específicamente a las competencias vinculadas a los rasgos de la personalidad (Alles, 2006). Ello evidencia una discrepancia entre el sector educativo y el sector empresarial sobre la finalidad de los procesos de formación, cuya repercusiones afectan la competitividad del país.

Sin embargo, la tendencia de estos procesos de formación a lo largo de la vida, consiste en la responsabilidad que asume la persona en cuanto a su formación, es decir, "un control individual considerable de lo que se aprende y de cuándo, dónde y cómo se imparte la formación" (Organización para la Cooperación y el Desarrollo Económico, OCDE, 2004, p. 22). De esta manera se van desplazando, desde las organizaciones laborales hacia las personas, la responsabilidad de mantener su lugar en el mercado de trabajo, por medio del uso del término de empleabilidad (De Pádua y Moyzes, 2004).

Estas situaciones de transformaciones descritas ponen de manifiesto la flexibilidad laboral como una de las tendencias de los mercados de trabajo, la cual requiere -de la persona trabajadora, la familia y la sociedad- la capacidad de adaptación a esta nueva exigencia.

No obstante, la flexibilidad laboral puede traer repercusiones a las personas, a las organizaciones e incluso a la sociedad, pues en los mercados de trabajo caracterizados por una alta flexibilidad, se da una mayor desprotección social; mientras que en los mercados con una alta protección social, su flexibilidad es mínima y limita su competividad a nivel global. Se manifiesta, así, una dualidad que requiere de la atención de diversos actores sociales.

Un aspecto vinculado con esta situación es la precarización laboral en la cual se promueven procesos de exclusión, desigualdad social entre las personas que tienen un trabajo de calidad y aquellas que no lo tienen (Alcover et al. 2004), potenciando, de esta manera, situaciones de inestabilidad en el desarrollo y gestión de las carreras de las personas, así como en las demás dimensiones de su vida.

Sin embargo, ante estas transformaciones sobre la concepción de las organizaciones, del trabajo y las implicaciones que conllevan en el desarrollo del país, se requiere de una fuerza laboral con bases muy sólidas en su educación científica-tecnológica y con capacidad de adquirir nuevas competencias a lo largo de la vida, asimismo que abracen la flexiseguridad ${ }^{2}$ de la sociedad del conocimiento (Gazier, 2006).

\footnotetext{
${ }^{2}$ La flexiseguridad hace referencia a un modelo económico de organización que busca lograr un equilibrio entre las exigencias del mercado laboral globalizado y la protección social a las personas trabajadoras.
} 
Pues, como señalan Buendía, Expósito y Sánchez (2012), "el desarrollo de un territorio queda determinado en cuanto a sus aspectos socioeconómicos y laborales, por la cualificación profesional y formativa de su población y su adaptabilidad a las necesidades de la demanda de empleo" (p. 67).

\section{La orientación laboral}

Ante la serie de transformaciones que experimentan los mercados de trabajo a nivel mundial y nacional, así como ante las nuevas exigencias que se les plantean a las personas para ingresar y mantenerse en el mercado de trabajo, la orientación como disciplina se ha visto en la necesidad de hacer replanteamientos desde lo ontológico, epistemológico y metodológico para dar respuesta a las nuevas exigencias económicas, sociales, organizacionales, culturales y tecnológicas (Echeverría, 2008; Enriques, 2007; Savickas, et al., 2009). En palabras de Bock (2010, citado por González, 2014):

El eje central de intervención de la orientación profesional ${ }^{3}$ debería ser modificado. En vez de tener como preocupación central la escogencia profesional de los sujetos, debería dedicarse a que ello comprendieran la organización y la forma de trabajo de la sociedad actual. (p. 27)

Por lo que"no podemos seguir pensando en una Orientación limitada al campo educativo y debemos ir pensando en otros tipos de contextos" (Gavilán 2006, citada por González 2014 p. 26). Lo que implicaría acercar a la orientación a otras corrientes epistemológicas que contemplen la relación dialógica entre el ser humano con su entorno y"permitan acceder a muchos aspectos de la vida de las personas en sus propios contextos" (Ribeiro, 2013, p. 4).

Así mismo, Enriques (2007) propone que, para dar respuesta a estos retos, la orientación debe "responder a lo Global/Internacional, responder a lo Organizacional, y responder a lo Individual y, en cada uno de esos niveles, reflejar los papeles de la teoría, de la investigación y de la intervención" (Enriques, 2007, p. 91).

En el país se han venido realizando esfuerzos muy valiosos para diversificar los contextos de intervención de la disciplina, entre ellos, se destaca el inicio de la formación de profesionales en orientación laboral en el 2012. Esta emerge en un contexto caracterizado por la desigualdad

${ }^{3}$ En esta discusión no se pretende establecer una equivalencia entre los conceptos de orientación profesional y de orientación laboral, pues el primero se encuentra contextualizado en el ámbito europeo, donde numerosos estudios han desarrollado su clarificación conceptual y han establecido su marco de acción a lo largo de la vida de las personas (ver OCDE, 2004). Por su parte, en el ámbito costarricense se ha empleado el concepto de orientación, desde el cual han surgido algunas especializaciones, entre ellas la orientación laboral. Sin embargo, se decide utilizar esta cita debido a la reflexión metodológica que realiza el autor sobre la disciplina. 
doi: http://dx.doi.org/10.15359/ree.21-2.19

URL: http://www.una.ac.cr/educare

CORREO: educare@una.cr

social, inestabilidad económica, altas tasas de desempleo, precarización laboral (en especial de grupos desfavorecidos en el mercado de trabajo), es decir, un contexto de incertidumbre laboral, en el que las personas se proponen alcanzar su desarrollo y bienestar desde su multidimensionalidad.

Asimismo, las autoras Ureña y Barboza (2015) destacan este hecho al mencionar que el área de intervención de la orientación (como disciplina), en Costa Rica, se ha ido diversificando, lo cual tradicionalmente se ha enfocado a lo educativo, pero que en la actualidad se logra identificar la intervención de profesionales de esta disciplina en el contexto sociolaboral. A partir de esta diversificación de los contextos interviene la orientación y dadas las demandas y necesidades de las personas en los entornos sociolaborales, que surge la orientación laboral en nuestro país.

De esta misma forma, Gavilán (2006) menciona como campo de acción de esta especialización:

Al estado en que se encuentran todas las actividades relacionadas con lo laboral: inserción, desocupación, precarización, inestabilidad, reconversión ... el desarrollo productivo, la inversión capital-trabajo, la proliferación y destrucción de ocupaciones, las nuevas ocupaciones, el desarrollo de nuevas competencias, la cultura del trabajo, los procesos de integración y desintegración de las ocupaciones, la legislación laboral y empresarial, y las políticas de intervención. (p. 23)

No obstante, al ser un aréa de especialización de la orientación relativamente nueva, no hay una conceptualización clara de esta, por lo que se requiere la construcción de una definición que permita describir su marco de acción. Cabe señalar que son escasas las fuentes bibliográficas que mencionan la orientación laboral como tal y más bien diversos autores emplean otros conceptos como orientación ocupacional, orientación para el empleo, orientación vocacional, los cuales podrían contribuir a un primer acercamiento conceptual. En la Tabla 1 se detallan estos conceptos, así como algunas características que facilitan una mayor comprensión.

Entre las características contempladas en la Tabla 1, se encuentra la población o poblaciones a las que se dirigen las intervenciones de las personas profesionales en orientación, la segunda característica consiste en identificar si estas intervenciones son de un carácter puntual o procesual (las intervenciones puntuales se realizan en momentos específicos, mientras que las intervenciones procesuales se realizan de forma continua y sistemática); asimismo, identificar si estas son reactivas o proactivas (las intervenciones reactivas se realizan una vez producido el problema, mientras que las intervenciones proactivas se realizan antes de que ocurra el problema). Y, por último, se identifican las modalidades de intervención individual y grupal en estas definiciones. 
Tabla 1: Aproximaciones conceptuales a la orientación laboral

\begin{tabular}{|c|c|c|c|}
\hline Definición & Población & $\begin{array}{l}\text { Características de } \\
\text { las intervenciones }\end{array}$ & $\begin{array}{c}\text { Modalidades de } \\
\text { intervención }\end{array}$ \\
\hline $\begin{array}{l}\text { Orientación para la carrera: Es un conjunto -preferiblemente } \\
\text { un programa sistemático- de procesos, técnicas o servicios } \\
\text { diseñados para ayudar al individuo a entender y actuar pro- } \\
\text { porcionándole un autoconocimiento y un conocimiento de } \\
\text { las oportunidades de trabajo, educación y tiempo libre y } \\
\text { ayudándole a desarrollar habilidades de toma de decisiones } \\
\text { para poder crear y dirigir el propio desarrollo de la carrera. } \\
\text { (Herr } 1982 \text { citado por Pereira, } 1995 \text { p. 314) }\end{array}$ & $\begin{array}{l}\text { Personas } \\
\text { de todas las } \\
\text { edades }\end{array}$ & $\begin{array}{l}\checkmark \text { Procesual } \\
\checkmark \text { Proactiva }\end{array}$ & $\begin{array}{l}\checkmark \text { Individual } \\
\checkmark \text { Grupal }\end{array}$ \\
\hline $\begin{array}{l}\text { Orientación vocacional: Es un proceso de maduración y apren- } \\
\text { dizaje personal a través del cual se presta una ayuda al individuo } \\
\text { para facilitarle la toma de decisiones vocacionales, con el objeto } \\
\text { de que logre un nivel óptimo de realización personal y de inte- } \\
\text { gración a través del mundo del trabajo. (Castaño López-Mesas, } \\
1983 \text { citado por Ceinos, } 2008 \text { p. 86) }\end{array}$ & $\begin{array}{l}\text { Personas } \\
\text { de todas las } \\
\text { edades }\end{array}$ & $\begin{array}{l}\checkmark \text { Procesual } \\
\checkmark \text { Proactiva }\end{array}$ & $\begin{array}{l}\checkmark \text { Individual } \\
\checkmark \text { Grupal }\end{array}$ \\
\hline $\begin{array}{l}\text { Orientación ocupacional: La orientación ocupacional nace } \\
\text { para dar respuesta a la problemática que surge en la relación } \\
\text { con el empleo y la formación para ocupar de forma más o me- } \\
\text { nos inmediata un puesto de trabajo. (Montané, 1993, p. 39) }\end{array}$ & $\begin{array}{l}\text { Personas en } \\
\text { búsqueda de } \\
\text { empleo }\end{array}$ & $\begin{array}{l}\checkmark \text { Puntual } \\
\checkmark \text { Reactiva }\end{array}$ & $\begin{array}{l}\checkmark \text { Individual } \\
\checkmark \text { Grupal }\end{array}$ \\
\hline $\begin{array}{l}\text { Orientación para el empleo: Es intervenir en la dificultad } \\
\text { de una persona para encontrar, mantener o progresar en un } \\
\text { empleo, ayudando en la adquisión de aquellas competencias } \\
\text { (técnicas, metodológicas, personales y participativas) que fa- } \\
\text { ciliten su inserción, reinserción, recualificación mediante una } \\
\text { atención individual y grupal interveniendo por programas } \\
\text { o servicios par aumentar su empleabilidad y ocupabilidad y } \\
\text { cuyas características esenciales son: carácter puntual y fun- } \\
\text { ción reactiva. Sarasola (2008, p. } 363 \text { ) }\end{array}$ & $\begin{array}{l}\text { Personas en } \\
\text { búsqueda de } \\
\text { empleo }\end{array}$ & $\begin{array}{l}\checkmark \text { Puntual } \\
\checkmark \text { Reactiva }\end{array}$ & $\begin{array}{l}\checkmark \text { Individual } \\
\checkmark \text { Grupal }\end{array}$ \\
\hline $\begin{array}{l}\text { Orientación laboral: Es un proceso continuo, sistemático, e } \\
\text { intencional que consiste en una serie de acciones programa- } \\
\text { das y estructuradas para facilitar que las personas en búsque- } \\
\text { da de empleo accedan en las mejores condiciones al merca- } \\
\text { do de trabajo. Se trata de un acompañamiento individual o } \\
\text { grupal en el que se facilita que las personas usuarias reflex- } \\
\text { ionen sobre su situación laboral, tomen decisiones sobre sus } \\
\text { proyectos ocupacionales y actúen de forma autónoma en la } \\
\text { búsqueda de empleo mediante la planificación de sus objeti- } \\
\text { vos laborales a corto, medio o largo plazo y de las actuacio- } \\
\text { nes para conseguirlo. (Sistema Nacional de Intermediación, } \\
\text { Orientación e Información de Empleo, SIOIE, 2010, p. 59) }\end{array}$ & $\begin{array}{l}\text { Personas en } \\
\text { búsqueda de } \\
\text { empleo }\end{array}$ & $\begin{array}{l}\checkmark \text { Puntual } \\
\checkmark \text { Reactiva }\end{array}$ & $\begin{array}{l}\checkmark \text { Individual } \\
\checkmark \text { Grupal }\end{array}$ \\
\hline
\end{tabular}


doi: http://dx.doi.org/10.15359/ree.21-2.19

URL: http://www.una.ac.cr/educare

CORREO: educare@una.cr

\begin{tabular}{|c|c|c|c|}
\hline Definición & Población & $\begin{array}{l}\text { Características de } \\
\text { las intervenciones }\end{array}$ & $\begin{array}{l}\text { Modalidades de } \\
\text { intervención }\end{array}$ \\
\hline $\begin{array}{l}\text { Orientación ocupacional: La orientación ocupacional cuya } \\
\text { atención va dirigida hacia "el aprendizaje de destrezas, intere- } \\
\text { ses, creencias, valores, hábitos de trabajo y cualidades perso- } \\
\text { nales que capacita a cada persona ... para crear una vida sat- } \\
\text { isfactoria en un mundo laboral constantemente cambiante" } \\
\text { (De León y Rodríguez, 2008, p. 10). }\end{array}$ & $\begin{array}{l}\text { Personas en } \\
\text { búsqueda de } \\
\text { empleo }\end{array}$ & $\begin{array}{l}\checkmark \text { Puntual } \\
\checkmark \text { Reactiva }\end{array}$ & $\begin{array}{l}\checkmark \text { Individual } \\
\checkmark \text { Grupal }\end{array}$ \\
\hline $\begin{array}{l}\text { Orientación laboral: La Orientación Laboral como rama de la } \\
\text { Orientación interviene en los sistemas humanos inmersos en } \\
\text { el contexto de las organizaciones: empresas, cooperativas, aso- } \\
\text { ciaciones o cualquier otra forma de organización laboral huma- } \\
\text { na. De esta manera centra su interés profesional en el desarrollo } \\
\text { de la autonomía y la efectiva funcionalidad de la persona en } \\
\text { sus roles laborales en consonancia con los objetivos de la orga- } \\
\text { nización para la cual presta sus servicios (Barreto, 2014, p. 427). }\end{array}$ & $\begin{array}{l}\text { Personas } \\
\text { trabajadoras }\end{array}$ & $\begin{array}{l}\checkmark \text { Procesual } \\
\checkmark \text { Proactiva }\end{array}$ & $\begin{array}{l}\checkmark \text { Individual } \\
\checkmark \text { Grupal }\end{array}$ \\
\hline
\end{tabular}

Nota: Elaboración propia.

A partir de la información de la Tabla 1 comparativa, se logra identificar que, en la mayoría de los conceptos, se establece una naturaleza reactiva y puntual de la orientación, es decir, las intervenciones se realizan cuando ocurre el problema. Además, se observa que, en la mayoría de los conceptos, el propósito de la orientación consiste en la inserción laboral. Asimismo, la población de atención la conforma las personas en búsqueda de empleo. Por último, se identifica en todos los conceptos las modalidades de intervención individual y grupal.

La mayoría de estos conceptos son enmarcados en el ámbito de la Unión Europea, donde la orientación es considerada como un eje central de las políticas activas de empleo (OCDE, 2004). Estas políticas tienen como objetivo "promover la inserción y el mantenimiento laboral de las personas" (Organización Internacional del Trabajo, OIT, 2012, p. 9). Esta misma instancia menciona que en las políticas activas de empleo se pueden identificar tres líneas de actuación:

a) Intermediación: comprende las acciones para facilitar la interracción entre la oferta y la demanda. Las principales actuaciones sobre las personas son las de orientación, información y seguimiento. Sobre la demanda se incide facilitando sus procesos de búsqueda de personal con las características adecuadas.

b) Formación para el empleo: comprende las medidas destinadas a facilitar que las personas accedan a una capacitación adecuada.

c) Promoción del empleo: esta línea de actuación tiene como objetivo la creación de nuevos empleos, asalariados o independientes. (p. 10) 
Estos conceptos presentados constituyen un primer acercamiento valioso a la orientación laboral, no obstante, definirla requiere de un proceso constante de construcción, deconstrucción y reconstrucción en función de sus objetivos y campos de acción. Debido a ello, para efectos de esta revisión bibliográfica, en la Tabla 2 se propone un acercamiento conceptual a la orientación laboral.

Tabla 2: Definición de orientación laboral

La orientación laboral es:

Un proceso sistemático, formativo dirigido a la persona trabajadora, con la finalidad, de facilitar en ella, las competencias que contribuyan a su desarrollo y bienestar laboral, mediante una intervención continua y sistémica de los procesos sociolaborales que interactúan entre esta y sus entornos.

¿Por qué es un proceso sistemático y formativo? La orientación laboral como un proceso sistemático y formativo hace referencia a una gama de acciones programadas y estructuradas, las cuales facilitan en las personas un aprendizaje experiencial para el desarrollo de sus competencias.

¿Por qué se dirige a la persona trabajadora?

Sus acciones se dirigen a la persona trabajadora, debido a que "la dimensión laboral-ocupacional ocupa un lugar trascendental en el desarrollo personal, económico y social de cualquier país" (UCR, s. f., p. 2).

¿Por qué facilita competencias para el desarrollo y bienestar laboral?
Debido a que la orientación laboral promueve el desarrollo de personas íntegras, integrales y competentes en entornos cambiantes. Las competencias desde un enfoque socioformativo se asumen como una dimensión más del desarrollo humano y son comprendidas como actuaciones integrales de las personas ante actividades y problemas del contexto con ... ética e idoneidad, en tanto articulan los saberes (saber ser, ... saber conocer, saber hacer) con el manejo de las situaciones externas del contexto, asumiendo los cambios y la incertidumbre con autonomía y creatividad. (Tobón, 2010, p. 25)

Esto significa que la orientación laboral debe actuar no solo en la persona sino que de forma recursiva y dialógica, debe actuar en los entornos inmediatos en que la persona interactúa de manera directa con los procesos laborales que conducen a su desarrollo y bienestar y con los entornos más amplios en el que la persona no interactúan de manera directa con estos procesos.

Nota: Elaboración propia. 
doi: http://dx.doi.org/10.15359/ree.21-2.19

URL: http://www.una.ac.cr/educare

CORREO: educare@una.cr

Esta definición busca dar un acercamiento epistemológico de la orientación laboral al pensamiento complejo, promoviendo de esta manera una visión global, compleja y sistémica de los procesos sociolaborales que interactúan de manera dialógica entre las personas y los entornos, poniendo como eje central el desarrollo del ser humano. El énfasis de esta conceptualización es fundamentada por la postura de Arguedas (2014), quien menciona que "el desarrollo humano tiene que seguir ocupando una posición central en el trabajo de la persona profesional en Orientación" (p. 261).

Para este fin, se requiere que las personas profesionales en orientación laboral tengan una sólida formación interdisciplinaria en la que integren el saber ser, con el saber conocer y el saber hacer, en el desarrollo de procesos de investigación y de intervención.

A partir de la construcción conceptual y de la revisión de los programas de los cursos: "Trabajo y Proyecto ocupacional I", "Desarrollo de Competencias Sociolaborales", "Desarrollo Organizacional", "Orientación Laboral I", "Orientación Laboral II", "Trabajo y Proyecto Ocupacional II" de la Maestría Académica en Educación con énfasis en Orientación Laboral (Universidad de Costa Rica, Sistema de Estudios de Posgrado, 2013), se visualizan algunos ejes generales en el marco de acción para las personas profesionales de la orientación laboral; entre estos se pueden mencionar:

Desde lo global

$\checkmark$ Análisis de las tendencias del mercado laboral

$\checkmark$ Estudios de prospección de empleo

$\checkmark$ Política de empleo

$\checkmark$ Atracción de inversión extranjera

$\checkmark$ Compresión del trabajo en la sociedad del conocimiento

Desde lo organizacional

$\checkmark$ Gestión del talento humano

$\checkmark$ Desarrollo organizacional

$\checkmark$ Calidad de la vida laboral-salud ocupacional

$\checkmark$ Responsabilidad social

$\checkmark$ Cultura y clima organizacional 
Desde lo individual

$\checkmark$ Procesos de búsqueda de empleo

$\checkmark$ Procesos de inserción, permanencia y retiro laboral

$\checkmark$ Asesoramiento para la carrera

$\checkmark$ Empleabilidad

$\checkmark$ Emprendimientos

$\checkmark$ Recreación y ocio

$\checkmark$ Desarrollo de competencias

$\checkmark$ Desempeño laboral

$\checkmark$ Trabajo voluntariado

Asimismo, a partir de esta aproximación teórica de la orientación laboral, se pueden proyectar algunos posibles escenarios laborales en el país para las futuras personas especialistas de esta área, entre ellas se pueden mencionar:

$\checkmark$ Asociaciones de desarrollo comunal

$\checkmark$ Centros educativos de formación profesional, colegios técnicos, universidades

$\checkmark$ Consejo Nacional de la Persona Joven

$\checkmark$ Consejo Nacional de Personas con Discapacidad

$\checkmark$ Ejercicio profesional privado

$\checkmark$ Ministerio de Trabajo y Seguridad Social

$\checkmark$ Observatorios laborales

$\checkmark$ Oficinas de atracción de inversión extranjera

$\checkmark$ Oficinas de incubación de empresas

$\checkmark$ Organización Internacional del Trabajo

$\checkmark$ Organizaciones laborales (instituciones públicas, cooperativas, empresas privadas, entre otras)

$\checkmark$ Organizaciones no gubernamentales

$\checkmark$ Servicios municipales de intermediación laboral

$\checkmark$ Sistema penitenciario 
doi: http://dx.doi.org/10.15359/ree.21-2.19

URL: http://www.una.ac.cr/educare

CORREO: educare@una.cr

\section{Conclusiones}

La orientación laboral es una especialización de la disciplina de la orientación que promueve una visión integral de los procesos sociolaborales que interactúan en la vida laboral de la persona y el entorno en el que esta se desarrolla. Por tanto, las personas especialistas requieren una sólida formación interdisciplinaria en la que integren el saber ser, con el saber conocer y el saber hacer, en el desarrollo de procesos de investigación y de intervención desde lo organizacional y lo individual.

Además, esta especialización enmarcada desde el pensamiento sistémico, comprende las organizaciones como sistemas vivos conformados por subsistemas interdependientes que interactúan entre sí y el entorno; enfatizando, de esta manera, en las interacciones entre la organización, las personas trabajadoras y el entorno que potencialicen su propio crecimiento, el de sus miembros y el de la comunidad.

Es importante enfatizar que el eje central de toda intervención desde la orientación laboral es el ser humano, por lo que se busca promover las competencias para el desarrollo y bienestar laboral de la persona trabajadora desde los diferentes entornos en la que esta interactúa y, a la vez, apoyar las organizaciones a ser lugares más productivos y con sentido humano, por medio de la creación de las condiciones en los entornos laborales que faciliten ese desarrollo y bienestar personal, y de la comunidad.

\section{Referencias}

Alcover, C. M., Martínez, D., Rodríguez, F. y Domínguez, R. (2004). Introducción a la psicología del trabajo. Madrid: McGraw-Hill.

Alles, M. A. (2006). Influencia de las caracteristicas de personalidad (competencias) en la empleabilidad de profesionales (Tesis de doctorado). Universidad de Buenos Aires, Argentina, Buenos Aires. Recuperado de http://bibliotecadigital.econ.uba.ar/download/ tesis/1501-1181 AllesMA.pdf

Arguedas, I. (2014). Desarrollo humano en todos sus ámbitos: Eje de la orientación. En Colegio de Profesionales en Orientación (Ed.), Memoria Primer congreso nacional de orientación "50 años de la orientación en Costa Rica: Logros y desafíos" (pp. 261-268). Heredia, Costa Rica: Colegio de Profesionales en Orientación. Recuperado de http://www.cpocr.org/wpcontent/uploads/2014/11/Memoria-Congreso-de-Profesinales-en-Orientacion-2014.pdf

Barreto, A. A. (2014). La orientación laboral en Latinoamérica del siglo 21. Capítulo Venezuela [Resumen]. En Colegio de Profesionales en Orientación (Ed.), Memoria Primer congreso nacional de orientación "50 años de la orientación en Costa Rica: Logros y desafíos" (pp. 427437). Heredia, Costa Rica: Colegio de Profesionales en Orientación. 
Buendía, L., Expósito, J.y Sánchez, M. (2012). La formación profesional para el empleo. Propuestas para el desarrollo local. REOP, 23(1), 66-84. Recuperado de http://e-spacio.uned.es/fez/ eserv/bibliuned:revistaREOP-2012-23-1-5030/Documento.pdf

Ceinos, M. C. (2008). Diagnóstico de las competencias de los orientadores laborales en el uso de las tecnologías de la información y comunicación (Tesis doctoral). Universidad de Santiago Compostela, España. Recuperado de https://minerva.usc.es/xmlui/bitstream/ handle/10347/2486/9788498871081 content.pdf? sequence=1\&isAllowed=y

David, P. A. y Foray, D. (2002). Una introducción a la economía y a la sociedad del saber. Revista Internacional de Ciencias Sociales, 171, 7-28. Recuperado de http://www.oei.es/historico/ salactsi/rics171.htm

De León y Rodríguez (2008). El efecto de la orientación vocacional en la elección de carrera. Revista Mexicana de Orientación Educativa, 5(13), 10-16. Recuperado de http://pepsic. bvsalud.org/pdf/remo/v5n13/v5n13a04.pdf

De Pádua, A. y Moyzes, A. (2004). Percurso semântico do tema empregabilidade: A (re)construção de parte da história de uma empresa de telefonia. $R A C, 8(1), 129-150$. Recuperado de http://www.scielo.br/pdf/rac/v8n1/v8n1a07.pdf

Echeverría, B. (Coord.). (2008). Orientación profesional. Barcelona: UOC.

Enriques, M. J. (2007). Desarrollo y gestión de carreras con adultos en el siglo XXI: Lecturas hacia una amornización de lo global y de lo individual. Revista Electrónica de Investigación Psicoeducativa, 5(1), 75-102. Recuperado de http://www.investigacion-psicopedagogica. com/revista/articulos/11/espannol/Art 11 159.pdf

Fundación Demuca. (2011). Políticas locales para la promoción del empleo. San José, Costa Rica: Autor. Recuperado de https://observatorioredesempresariales.files.wordpress. com/2011/07/politicas-locales-promocion-empleo-demuca.pdf

Gallardo, A. (2002). La era de la incertidumbre, la organización y la teoría del caos. Administración y Organizaciones, 4(8), 63-76. Recuperado http://gceduc.wikispaces.com/file/view/ $\underline{\text { La+era+de+la+incertidumbre.pdf }}$

Gavilán, M. (Julio, 2006). La formación de orientadores en contextos complejos con especial referencia a Latinoamérica. Conferencia inaugural presentada en I Congreso Latinoamericano de Formadores de Orientadores y III Congreso Venezolano Interdisciplinario de Orientación "La formación del orientador y la nueva concepción de la educación". Universidad de Carabobo, Valencia, Venezuela. Recuperado de https://es.scribd.com/ document/17082525/Formacion-Orientadores\# 
doi: http://dx.doi.org/10.15359/ree.21-2.19

URL: http://www.una.ac.cr/educare

CORREO: educare@una.cr

Gazier, B. (Mayo, 2006). Flexicurity and social dialogue, European ways. Ponencia presentada en DG EMPL Seminar on Flexicurity (pp. 1-19). París: University Paris 1 y CNRS. Recuperado de http://www.metiseurope.eu/content/pdf/flexicurite/4 gazier.pdf.pdf

González, J. (2014). La orientación en América Latina: Recuento histórico y principales desafíos [Conferencia]. En Colegio de Profesionales en Orientación (Ed.), Memoria Primer congreso nacional de orientación "50 años de la orientación en Costa Rica: Logros y desafíos" (pp. 2533). Heredia, Costa Rica: Colegio de Profesionales en Orientación.

Guzmán, M. (2007). La anticipación de la crisis (Una aplicación del enfoque del caos). EIDOS, 7, 128-159. Recuperado de https://dialnet.unirioja.es/servlet/articulo?codigo $=2361663$

Marín, A. (2011). Propuesta de un programa de capacitación dirigido a disminuir la brecha entre las competencias laborales establecidas por la institución como óptimas para el desempeño del puesto y las competencias laborales identificadas en la población del Banco Popular (Tesis de maestría). Universidad de Costa Rica, San José.

Maslow, A. H. (1991). Motivación y personalidad. Madrid: Ediciones Díaz de Santos.

Montané, J. (1993). Orientación ocupacional. Barcelona: Ediciones CEAC.

Morin, E. (1990). Introducción al pensamiento complejo. Barcelona: Gedisa.

Morin, E. (1999). Los siete saberes necesarios para la educación del futuro. Francia: UNESCO.

Organización Internacional del Trabajo (OIT). (Agosto, 2012). Curso regional de la intermediación y orientación laboral (Manual del curso). San José: Costa Rica: PRODOC.

Organización para la Cooperación y el Desarrollo Económico (OCDE). (2004). Orientación profesional y políticas públicas. Cómo acortar distancias. París: Autor. Recuperado de http:// www.oecd.org/edu/innovation-education/34529291.pdf

Pereira, M. (1995). La fundamentación teórica de la educación para la Carrera. Revista Educación, 307, 309-338. Recuperado de http://www.mecd.gob.es/dctm/revista-de-educacion/ articulosre307/re3071300495.pdf?documentld=0901e72b81272e9d

Rentería, E. (2008). Empregabidade: Versôes e implicaçôes. Uma leitura desde a psicologia social (Tesis doctoral). Universidades de Sâo Paulo, Sâo Paulo, Brasil.

Rentería, E., García, E., Restrepo, I. C. y Riascos, W. A. (2007). Sentidos del trabajo a partir de trayectorias y recursos personales para afrontar el mundo del trabajo. Perspectivas en psicología, 10, 127-151. 
Ribeiro, M. A. (2013). Reflexiones epistemológicas para la orientación profesional en América Latina: Una propuesta desde el construccionismo social. Revista Mexicana de Orientación Educativa, 10(24), 2-10. Recuperado de http://pepsic.bvsalud.org/pdf/remo/v10n24/a02.pdf

Sánchez, M. F. (2003). Las características del mercado de trabajo actual y el papel de la orientación profesional. En A. Sebastián (Coord.), Orientación profesional: Un proceso a lo largo de la vida (pp. 216-254). Madrid: Dykinson.

Sarasola, L. (2008). Cap. 6. Formación y orientación para el empleo. En B. Echeverría (Coord.), Orientación profesional (pp. 301-368). Barcelona: UOC.

Savickas, M. L., Nota, L., Rossier, J., Dauwalder, J.-P., Duarte, M. E., Guichard, J., ... Van Vianen, A. E. M. (2009). Life designing: A paradigm for career construction in the 21st century. Journal of Vocational Behavior, 75(3), 239-250. doi: http://dx.doi.org/10.1016/j.jvb.2009.04.004

Sistema Nacional de Intermediación, Orientación e Información de Empleo (SIOIE).(2010).Manual del servicio público de empleo de la República de Costa Rica. San José: Autor. Recuperado de https://es.scribd.com/document/96534559/Sistema-Nacional-de-IntermediacionOrientacion-e-Informacion-de-Empleo-SIOIE

Tobón,S.(2008).Laformación basadaencompetenciasen laeducación superior:Elenfoquecomplejo. Bogotá: Instituto Cife. Recuperado de http://cmapspublic3.ihmc.us/rid=1LVT9TXFX1VKC0TM-16YT/Formaci\%C3\%B3n\%20basada\%20en\%20competencias\%20(Sergio\%20 Tob\%C3\%B3n).pdf

Tobón, S. (2010). Formación integral y competencias. Pensamiento complejo, currículo, didáctica y evaluación. Bogotá: Ecoe Ediciones.

Universidad de Costa Rica. (s. f.). Programa de Investigación. Posgrado en Orientación. San José, Costa Rica: Autor. Manuscrito inédito.

Universidad de Costa Rica, Sistema de Estudios de Posgrado. (2013). Maestría Académica en Educación con énfasis en orientación laboral (Plan de Estudios). San José, Costa Rica: Autor.

Ureña, V. y Barboza, C. (2015). Aportes de la orientación vocacional en el contexto laboral. Revista Electrónica Actualidades Investigativas en Educación, 15(1), 1-21. doi: http://dx.doi. org/10.15517/aie.v15i1.17629 